\title{
Nerve growth factor receptor immunostaining suggests an extrinsic origin for hypertrophic nerves in Hirschsprung's disease
}

\author{
H Kobayashi, D S O'Briain, P Puri
}

\begin{abstract}
The expression of nerve growth factor receptor in colon from 20 patients with Hirshsprung's disease and 10 controls was studied immunohistochemically. The myenteric and submucous plexuses in the ganglionic bowel and hypertrophic nerve trunks in the aganglionic bowel displayed strong expression of nerve growth factor receptor. The most important finding was the identical localisation of nerve growth factor receptor immunoreactivity on the perineurium of both hypertrophic nerve trunks in Hirshsprung's diseases, and of normal extrinsic nerves, seen as a thick ring surrounding the nerve trunks. This suggests that the hypertrophic nerve trunks are of extrinsic origin and that nerve growth factor receptor plays a role in their development and maintenance.

(Gut 1994; 35: 1605-1607)
\end{abstract}

Hirshsprung's disease is characterised histologically by the absence of ganglion cells in the myenteric and submucous plexuses and the presence of hypertrophied nerve trunks in the space normally occupied by the ganglion cells. Although the bowel dysfunction in this disorder is considered to be produced by abnormalities of enteric nerves, the pathophysiological basis of the disease is not fully understood. There is no explanation for the occurrence of hypertrophied nerve trunks in the myenteric and submucous plexuses. Nerve growth factor (NGF) and its receptor (nerve growth factor receptor (NGFR)) are neurotrophic proteins that play an essential role in the normal development and survival of neurons in the peripheral and central nervous systems. ${ }^{1}$ During development, NGF is necessary for outgrowth of axons and establishment of synapses, and NGFR is the transmembrane protein that binds NGF and brings it into the cell. ${ }^{2}$ Cholinergic nerve hyperplasia is a consistent finding in the aganglionic segment of bowel. Recent studies have shown that the effect of NGF on the development and integrity of neurons is predominantly confined to the cholinergic system, and that the effects of NGF are transmitted via receptors localised within the cholinergic neurons. ${ }^{3-5}$ This study aimed to investigate the pattern of NGFR immunoreactivity in the normal and aganglionic colon.

Methods

The entire resected specimen of colon was obtained from 20 patients (aged 2 weeks to 13 years) with Hirshsprung's disease after Swenson's operation. Colonic tissues taken during closure of colostomy for imperforate anus (eight patients) and during bladder augmentation (two patients) acted as controls.

\section{NGFR IMMUNOHISTOCHEMISTRY}

Specimens were embedded in objective compound tissue and snap frozen. The tissue blocks were kept in $-70^{\circ} \mathrm{C}$ until processing. The tissue sections $(10 \mu \mathrm{m})$ were transferred to gelatin coated slides and dried for 20 minutes. They were then fixed by cold $\left(4^{\circ} \mathrm{C}\right)$ acetone for 10 minutes and stained by the avidin-biotin immunoperoxidase technique with monoclonal antibody to human NGFR (1:10 dilution, Boehringer Mannheim). Negative controls included substitution of non-immune serum or irrelevant antibodies for anti-NGFR.

\section{Results}

NORMAL BOWEL AND GANGLIONIC SEGMENT OF HIRSHSPRUNG'S DISEASE

There was strong NGFR staining of nerve fibres and ganglion cells of the submucosal and myenteric plexuses (Figure A). Numerous NGFR positive nerve fibres were present in the circular as well as the longitudinal smooth muscle layers. Serosal (mesenteric) nerves showed prominent staining of a thick perineurial layer in addition to staining of the nerve fibres (Figure B). This pattern of the perineurial staining seemed to be confined to extrinsic nerves as it was not present within the nerves of the muscle or submucous layer.

\section{AGANGLIONIC SEGMENT OF HIRSHSPRUNG'S} DISEASE

Minimal NGFR activity could be detected in the nerves within the circular and longitudinal muscle of aganglionic colon. The most striking finding was the localisation of NGFR immunoreactivity on the perineurium of hypertrophic nerve trunks, seen as a thick ring surrounding the abnormal nerve trunks (which also stained) in both submucous and myenteric plexuses of aganglionic segment of colon (Figure $\mathrm{C}$ ) and resembling the pattern seen in the serosal (mesenteric) nerves of the normal and ganglionic Hirshsprung's disease colon (Figure B). 


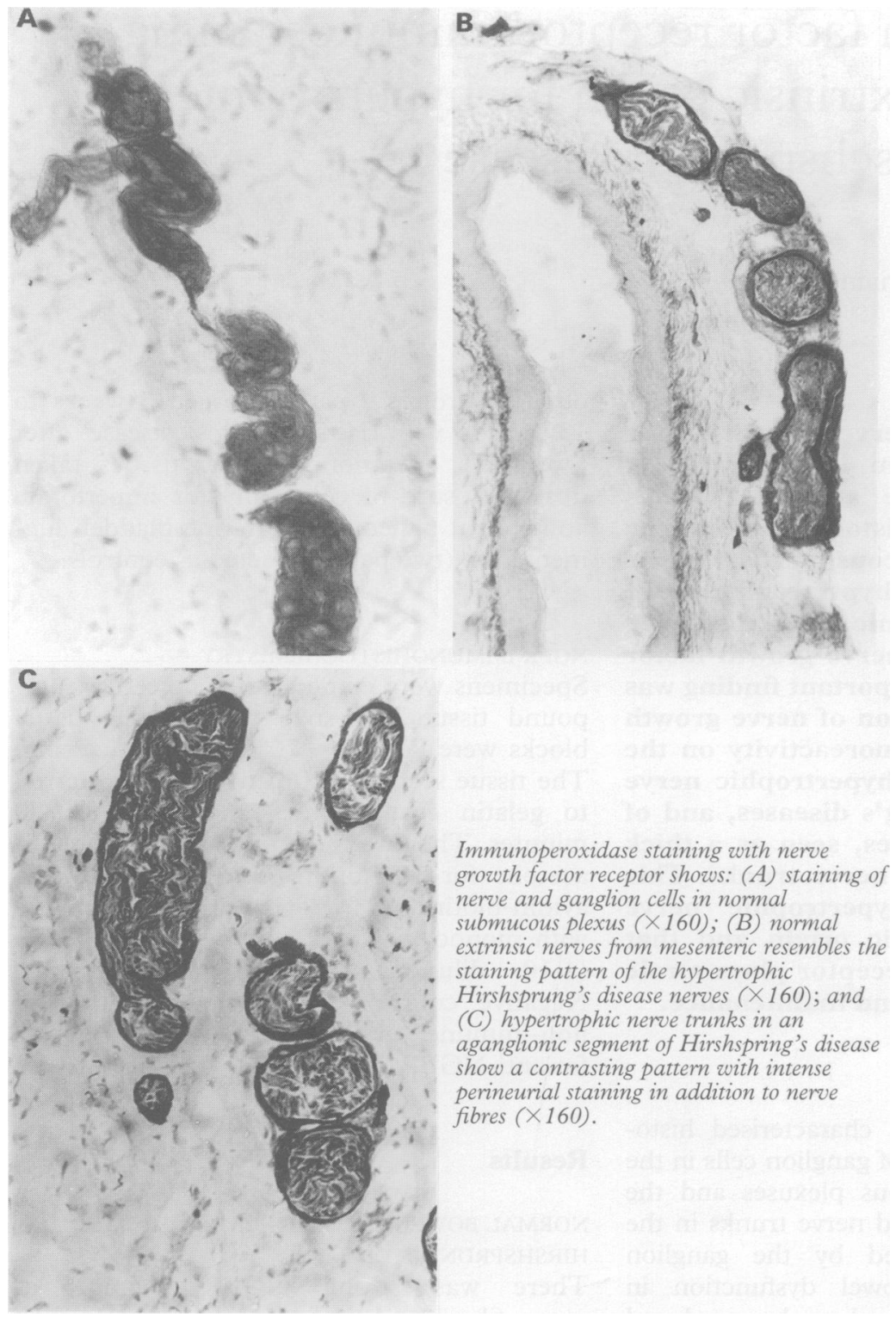

Discussion

Immunohistochemical staining of colon from patients with Hirshsprung's disease showed the virtual absence of NGFR immunoreactivity in the circular and longitudinal muscle layers of aganglionic colon, whereas numerous NGFR positive nerve fibres were present in the smooth muscle layers of ganglionic colon. These results parallel findings of absent nerve fibres in the smooth muscle of Hirshsprung's disease using various other neuronal markers such as NADPH-diaphorase, neural cell adhesion molecule, S-100 protein, neuronspecific enolase, neurofilament protein, glial fibrillary acidic protein, and $\mathrm{D}_{7}$ monoclonal antibody. 67

The most striking finding in this study was the strong expression of NGFR immunoreactivity on the perineurium of hypertrophic nerve trunks in the submucous and myenteric plexuses. Although NGFR immunoreactivity was present in the nerve fibres of the hypertrophic trunks, the perineurium was the region in which NGFR expression constituted most reactivity in the form of a thick ring surrounding the hypertrophic nerve trunk. This seems to be the first demonstration of perineural staining in hypertrophic nerve trunks using monoclonal antibody to NGFR. Perineural staining of hypertrophic nerve trunks in Hirshsprung's disease has not been reported with other neuronal markers.

Many investigators have attempted to determine the nature and origin of the hypertrophic nerve trunks found in the aganglionic segment of colon in patients with Hirshsprung's disease. ${ }^{8-11}$ It has been suggested that hypertrophic nerve trunks originate from intrinsic nerves and that the abnormality of nerve hypertrophy in aganglionic segments is due to an increased number of nerve fibres rather than to an increase in their size. ${ }^{10}$ In contrast, whole-mount studies have suggested that the hypertrophic nerve trunks are blind ending, bulbous terminations of extrinsic nerves. ${ }^{11}$ Our results clearly show that the pattern of NGFR perineural staining of hypertrophic nerve trunks in the aganglionic segment was identical to that seen in the serosal (mesenteric) nerves of the bowel. The strong NGFR perineural staining of serosal (mesenteric) nerves in the normal bowel was confined only to extrinsic nerves and was not present in the nerves of the muscle or submucous layer. This suggests that the hypertrophic, nerve trunks originate from extrinsic enteric nerves.

The functional importance of this finding is unclear. In the normal colon direct innervation of muscle is provided by the intrinsic nervous system. The action of this system is modulated by the extrinsic nervous system (predominantly composed of cholinergic fibres from the sacral parasympathetic system) through its synaptic connections with the ganglia of the intrinsic system. In Hirshsprung's disease the ganglia are absent and the intrinsic innervation is noticeably distorted with an appreciable reduction in intramuscular nerves but an increase in intramuscular cholinergic fibres. The extrinsic nerves, in the absence of ganglia, fail to connect with the intrinsic system and become hypertrophic, ${ }^{11}$ carrying a thickened perineurium into the bowel submucosa. This hypertrophy contrasts with the finding in other sites $^{3}$ that during neural development fibres that fail to form synapses become atrophic, and suggests that there is a continuing trophic stimulous to the extrinsic nerves. Clearly NGF and its receptor play a role in this stimulus, possibly from NGF produced by the hypoinnervated colonic muscle or from the distorted intrinsic nerve plexuses. This study suggests that the hypertrophied nerve fibres in Hirshsprung's disease are of extrinsic origin and are a dynamic marker of the failure of integration of the intrinsic and extrinsic enteric neuronal systems in this condition.

1 Barde YA. Trophic factors and neuronal survival. Neuron 1989; 2: 1525-34

2 Thoenen $\mathrm{H}$, Barde YA. Physiology of nerve growth factor. Phys Rev 1980; 60: 1284-335.

3 Hefti F, Hartikka J, Salvatierra A, Weiner WJ, Mash DC. Localization of nerve growth factor receptors in cholinergic neurons of the human basal forebrain. Neurosci Lett 1986; 69: 37-41.

4 Kordower JH, Bartus RT, Bothwell M, Schatteman G, Gash DM. Nerve growth factor receptor immunoreactivity in the nonhuman primate (Cebus apella): distribution, morphology, and colocalization with cholinergic enzymes. F Comp Neurol 1988; 277: 465-86. 
5 Koliatsos VE, Clatterbuck RE, Nauta HW, Knusel B, Burton LE, Hefti FF, et al. Human nerve growth factor prevents degeneration of basal forebrain cholinergic neurons in primates. Ann Neurol 1991; 30: 831-40.

6 Kobayashi H, O'Briain DS, Puri P. Lack of expression of NADPH-diaphorase and neural cell adhesion molecule (NCAM) in colonic muscle of patients with Hirshsprung's disease. F Pediatr Surg 1994; 29: 301-4.

7 Fujimoto T, Reen DJ, Puri P. Immunohistochemical characterisation of abnormal innervation of colon in Hirshsprung's disease using $\mathrm{D}_{7}$ monoclonal antibody. f Pediatr Surg 1987; 22: 246-51.

8 Okamoto E, Satani M, Kuwata K. Histologic and embryologic studies on the innervation of the pelvic viscera in patients with Hirshsprung's disease. Surg Gynecol Obstet 1982; 155: 823-8.

9 Mackenzie JM, Dixon MF. An immunohistochemical study of the enteric neural plexi in Hirshsprung's disease. Histopathology 1987; 11: 1055-66.

10 Deguchi E, Iwai N, Goto Y, Yanagihara J, Fushiki S. An immunohistochemical study of neurofilament and microtubule-associated tau protein in the enteric innervation in Hirshsprung's disease. F Pediatr Surg 1993; 28: 886-90.

11 Tam PKH, Boyd GP. Origin, course and endings of abnormal enteric nerve fibres in Hirshsprung's disease Surg 1990; 25: 457-61. 